\title{
Evaluar la competencia mediática: una aproximación crítica desde las perspectivas pedagógica, política y metodológica
}

\section{Assessing media competence: a critical approach from the pedagogical, political and methodological perspectives}

\author{
Mateus, J. C., Andrada, P. y Ferrés, J. ${ }^{1}$ \\ Recibido: 14-05-2019 - Aceptado: 12-08-2019 \\ DOI: https://doi.org/10.26441/RC18.2-2019-A14
}

\begin{abstract}
RESUMEN: Este artículo argumenta la importancia de desarrollar un instrumento que mida la competencia mediática, esto es, la suma de capacidades para interactuar críticamente con los medios de comunicación. Presenta la problemática de la educación mediática en la sociedad de la información y aborda la necesidad de evaluar las capacidades de la ciudadanía en este contexto. Esta necesidad se aborda desde tres ámbitos: el desafío pedagógico que supone el poco conocimiento de la competencia mediática y su escasa formalización institucional; el discurso político que privilegia la tecnología educativa por encima de la educación mediática; y la cuestión metodológica, analizada a partir de las mediciones recientes sobre competencia mediática en España, Chile y Perú, en las que prima la autopercepción por encima del dominio real de las capacidades. Finalmente, se propone la construcción de un cuestionario en torno a dos cualidades: que sea integrador en cuanto a los contenidos e innovador en cuanto a la metodología.
\end{abstract}

Palabras clave: competencia mediática; medición; educación mediática; metodología.

\begin{abstract}
This article addresses the importance of developing an instrument that measures media competence, that is, the set of abilities to interact critically with the media. It introduces the problem of media education in the information society and argues the need to assess the skills of citizens in this context. It approaches this need from three perspectives: the pedagogical challenge that implies the little knowledge of the media competence and its little institutional formalization; the political discourse that privileges educational technology over media education; and the methodological question, analyzed from recent assessments of media competence applied in Spain, Chile and Peru, limited to the selfperception. Finally, we propose the construction of an instrument involving two features: integrative in terms of content, and innovative in terms of methodology.
\end{abstract}

Keywords: media competence; measurement; media education; methodology.

\footnotetext{
1 Julio-César Mateus es Doctor en Comunicación, Máster en Estudios Avanzados en Comunicación Social por la Universitat Pompeu Fabra y Profesor e investigador de la Facultad de Comunicación de la Universidad de Lima. jmateus@ulima.edu.pe, https://orcid.org/0000-0001-5161-3737
}

Pablo Andrada es Doctor en Comunicación por la Universitat Pompeu Fabra, Magister en Ciencias Sociales con mención en Sociología de la Modernización por la Universidad de Chile y Profesor de la Facultad de Economía, Gobierno y Comunicaciones de la Universidad Central de Chile. pabloandradasola@gmail.com, https://orcid.org/0000-0002-2887-5517

Joan Ferrés es Doctor en Ciencias de la Información y maestro. Fue Profesor en los Estudios de Comunicación Audiovisual e Investigador del Departamento de Comunicación de la Universitat Pompeu Fabra de Barcelona. joan.ferres@upf.edu, https://orcid.org/0000-0001-8995-6735 


\section{Introducción}

La educación mediática es un campo de estudio que surge en la década de los 70 debido al interés de la Organización de las Naciones Unidas para la Educación, la Ciencia y la Cultura, UNESCO, como una vía de superar el subdesarrollo y estimular la conciencia crítica de los ciudadanos (Buitrago, 2014).

En 1973 el International Council for Film, Television and Audiovisual Communication (ICFT) de la UNESCO (1977) definió la educación mediática como el estudio, aprendizaje y enseñanza de y sobre los medios modernos de comunicación, constituyendo un área de conocimiento autónoma que forma parte de la teoría y práctica educacional, y se vincula con los avances tecnológicos (p. 3). Desde entonces, la educación mediática plantea superar el uso de los medios como herramientas de soporte de otras áreas curriculares y se constituye por derecho propio en una línea de investigación en el campo de la Comunicación Social.

En 1982 un grupo de educadores, comunicadores e investigadores convocados por la UNESCO firmaron la Declaración de Grünwald, reconocida como el acta de nacimiento del movimiento mundial de la educación mediática. En el documento los expertos lamentaron la poca preocupación de la escuela por el tema, denunciando "un verdadero abismo [que] separa las experiencias educacionales que proponen estos sistemas [formal e informal] y el mundo real" (p. 1). Las razones para educar mediáticamente, que ya eran 'imperiosas' en los 80, pasaron a ser 'avasalladoras' en la actualidad debido al desarrollo tecnológico y al impacto de la mediatización en la vida cotidiana de las personas (Hjarvard, 2016).

El desarrollo de la educación mediática y su evaluación enfrentan al menos tres problemas que abordamos en este artículo: uno pedagógico, otro político institucional y el tercero de corte metodológico. Por un lado, la enseñanza sobre los medios no se encuentra formalizada en la mayoría de los currículos nacionales, a diferencia de otros campos del conocimiento. A pesar de que existe un consenso en relación al peso de los medios de comunicación en los aprendizajes y la socialización de las personas, no hay un acuerdo referido a la forma de definir qué es la competencia mediática. En este trabajo apostamos por una propuesta integradora que reconoce las prácticas comunicativas de manera convergente, es decir, incluyendo los medios tradicionales y los digitales de más reciente aparición (Ferrés y Piscitelli, 2012).

A nivel político institucional apreciamos que la mayoría de los estados han afrontado la relación entre la escuela y los medios desde un enfoque que privilegia la compra y el uso de tecnología. Las políticas educativas se han focalizado en dotar de computadoras y tablets a las escuelas públicas con el propósito de disminuir brechas tecnológicas. Esta opción se realiza desde una perspectiva productivista que postula una educación tecnológica que serviría para mejorar la fuerza laboral. Desde una perspectiva sociocultural, una perspectiva alternativa es la de reconocer la formación en medios como un derecho para el ejercicio de la ciudadanía.

En este contexto de digitalización y presencia ubicua de nuevos medios han aparecido también nuevos problemas sociales vinculados con la gestión de la información, como el tema de las noticias falsas, lo que ha vuelto a llamar la atención sobre este campo.

En los países desarrollados se ha vinculado más a la enseñanza crítica de los medios en el aula, en tanto en Latinoamérica ha estado vinculada a la pedagogía liberadora, la comunicación popular y los estudios culturales bajo la impronta de la educomunicación (Mateus y Quiroz, 2017). Partimos de la premisa de que la educación mediática, a pesar de su importancia y sus diversos enfoques, tiene un desarrollo asimétrico en el mundo. Mientras diversos países de la Unión Europea trabajan desde hace años en la implementación de un marco de actuación común, otros, como los iberoamericanos, no han logrado materializar iniciativas o políticas públicas que demuestren suficiente interés. 
A pesar de la asimetría señalada, encontramos dos constantes: el aumento de la inversión en infraestructura tecnológica con fines educativos y la preocupación por mejorar los niveles de interacción crítica de las personas con los medios. Resulta sintomático, al respecto, la preocupación mundial contemporánea por las noticias falsas como reto educativo en el mundo (Comisión Europea, 2018).

En una investigación del Instituto de Tecnología de Massachusetts (MIT) sobre el flujo del contenido de noticias en Twitter entre 2016 y 2017 se comprobó que las personas prefieren las noticias falsas y que estas se difunden más rápido que las noticias verdaderas (Vosoughi, Roy, y Aral, 2018). Otro estudio realizado en 27 países, que incluyen a Perú y Chile, mostró que la mayoría de las personas (65\%) percibe que las noticias falsas, las burbujas de filtro y la posverdad afectan más a otras personas que a ellas mismas, lo que pone de manifiesto una falsa autopercepción de competencia mediática e invulnerabilidad. En el mismo estudio, la mayoría señaló que veía regularmente noticias falsas, aunque más de la mitad aseguró haberlas creído en un primer momento (Ipsos, 2018).

El auge de la cultura digital y la preocupación de organismos multinacionales por las competencias digitales, mediáticas e informacionales han renovado la importancia de la formación docente como un hito para asegurar el desarrollo de capacidades en esta materia. En el año 2011 la UNESCO publicó el Curriculum para Profesores sobre Alfabetización Mediática e Informacional. Según este documento, educar en el contexto de la sociedad de la información implica atender la competencia informacional. Así también, educar en un entorno social en el que la mayor parte de comunicaciones son mediadas por tecnologías a través de viejas y nuevas prácticas comunicativas, exige atender la educación mediática y la capacidad de gestionar todas las dimensiones implicadas en la interacción con los medios.

A nivel académico, encontramos inestabilidad sobre los objetos de estudio, que buscan ir a la par de la acelerada renovación tecnológica y limitan su interés de manera exclusiva hacia los nuevos medios. En esa línea, identificamos un relato 'tecnosolucionista', impulsado por empresas privadas y gobiernos, que centra su atención en los dispositivos y en la formación instrumental, desatendiendo otras dimensiones propias de la educación mediática. Es decir, muchos pretenden solucionar el problema de la interacción ciudadano-medios centrándose en lo tecnológico (mejorar los algoritmos para detectar información falsa, por ejemplo) y no en el desarrollo de una competencia mediática que brinde autonomía a la persona.

A partir de estas premisas planteamos una reflexión que aporte a la construcción de un instrumento adecuado para evaluar los conocimientos, habilidades y actitudes en las personas en un escenario internacional. En suma, el objetivo de este artículo es argumentar la necesidad de desarrollar un instrumento que mida la capacidad de las personas para interactuar críticamente con los medios.

\section{Desarrollo: ¿Cómo se miden las competencias mediáticas?}

\subsection{El enfoque de competencias}

El enfoque de competencias es el resultado de una serie de evoluciones en la forma de comprender, organizar y evaluar el conocimiento, como bien narra Jean-Marie De Ketele (2008). En la antigüedad el conocimiento suponía el estudio de los textos de autores clásicos y las leyes que gobernaban la naturaleza. Luego el movimiento enciclopedista permitió especializar el conocimiento: el acceso a los descubrimientos e inventos empezó a multiplicarse y segmentarse, de manera que era imposible para una sola persona conocer todo. Desde entonces la educación formal empezó a compartimentar y jerarquizar aquellos conocimientos más importantes, creando inventarios para ser transmitidos de acuerdo a los programas por los 
que se optaba. Posteriormente, con el nacimiento del "espíritu científico" y la ilustración, el conocimiento empezó a definirse como la demostración del dominio sobre cierto asunto, plasmado en un objetivo cuyo cumplimiento debía ser observable. Esto traía, por cierto, la racionalización y secuenciación de procedimientos repetibles que puedan ser enseñados, aprendidos y evaluados. La educación se convirtió, de pronto, en un repertorio de objetivos jerarquizados que fueron operacionalizados y dispuestos en índices a partir de los cuales se diseñaron los programas de aprendizaje.

Un movimiento más reciente, de segunda mitad del siglo XX, es el de las competencias. Muy vinculado con la globalización de la economía, este movimiento dio lugar a una preocupación mayor por la eficiencia y el rendimiento, aliados de la competitividad y la rentabilidad (Ferrés y Piscitelli, 2012). Los centros de producción empezaron a crear sus propios servicios de formación para asegurar la 'competencia' de su mano de obra. Esta dinámica vinculada con la salida laboral caló en el ámbito escolar, que adoptó este enfoque como eje del nuevo sistema basado en la precisión de aquellas capacidades requeridas por las fuerzas del mercado y, en menor medida, por la sociedad. Este modelo provoca resistencias y polémicas, algunas de las cuales incluyen la discusión de ciertos contenidos, como la enseñanza de la filosofía en las escuelas o del sentido humanista de los estudios superiores (Guzmán Marín, 2017).

Existe un movimiento contemporáneo que reclama un esquema de formación más holístico y que cuestiona la 'dictadura' de las evaluaciones estandarizadas globales, como la prueba PISA (The Guardian, 2014). Lo actual, sin embargo, es que la gran mayoría de nuestros sistemas educativos están basados en competencias, lo que nos obliga a repensar, bajo este enfoque, si queremos que la educación mediática sea parte del sistema formativo.

\subsection{La justificación de lo mediático}

La premisa subyacente que justifica el desarrollo de una competencia mediática es que vivimos una sociedad mediatizada y, por lo tanto, la escuela debe contribuir a desarrollar capacidades para interactuar con el cambiante ecosistema mediático. De hecho, muchas de estas capacidades vienen siendo aprendidas extraescolarmente (Scolari, 2018), lo que resulta un gasto inútil para el sistema escolar, que bien podría aprovecharlas.

A diferencia de la tecnología educativa, que se ocupa de la mediación, la educación mediática se sostiene en la teoría de la mediatización. La primera se vincula al acto comunicativo producido por un medio, mientras que la mediatización se interesa por los cambios estructurales entre diferentes ámbitos culturales y sociales. Para Stig Hjarvard (2016), uno de los teóricos de la mediatización:

El funcionamiento de los medios no está sólo determinado por la tecnología, sino que también se caracteriza por prácticas simbólicas y estéticas (p. ej. géneros y convenciones estilísticas) y por normas institucionales (p. ej. legislación, mecanismos de mercado y estructuras organizacionales) [...] La mediatización debe entenderse como un proceso común de la alta modernidad, equiparable a otros procesos similares, como la urbanización, la globalización o la individualización (p. 239).

\subsection{La evaluación de la competencia}

En los últimos años se han elaborado diferentes instrumentos para medir la competencia mediática. Destacan la Evaluación de la Competencia Mediática de la ciudadanía española (Ferrés, García Matilla, Aguaded, Fernández Cavia, Figueras y Blanes, 2011) y los cuestionarios virtuales generados a partir del proyecto de Investigación y Desarrollo (I+D), La 
competencia en comunicación audiovisual en un entorno digital. Diagnóstico de necesidades en tres ámbitos sociales los profesionales de la comunicación, el ámbito universitario y el ámbito de la educación obligatoria, de los mismos autores.

En ambos proyectos se utilizan preguntas donde los sujetos dan cuenta de sus conocimientos, actitudes y habilidades ante los medios. La mayoría de los cuestionarios utilizan un método de autopercepción tomando como base las dimensiones de la competencia mediática (Ferrés, 2006 y 2007; Ferrés y Piscitelli, 2012), permitiendo conocer qué es lo que las personas dicen saber o hacer al relacionarse con los medios.

Aunque diversas instancias internacionales han convenido en la urgencia de establecer una competencia clave para todos los ciudadanos vinculada a los medios, su conceptualización es aún discutible. En 2006, por ejemplo, el Parlamento Europeo incluyó la competencia digital como una de las competencias básicas para el aprendizaje permanente. En España se adoptó esta resolución mediante los reales decretos 1513/2006, de 7 de diciembre, 1631/2006, de 29 de diciembre:

Esta competencia consiste en disponer de habilidades para buscar, obtener, procesar y comunicar información, y para transformarla en conocimiento. Incorpora diferentes habilidades, que van desde el acceso a la información hasta su transmisión en distintos soportes una vez tratada, incluyendo la utilización de las tecnologías de la información y la comunicación como elemento esencial para informarse, aprender y comunicarse (Ministerio de Educación, Cultura y Deporte de España, 2018).

También instituciones como la Organización para la Cooperación y el Desarrollo Económicos (OCDE), creadora e impulsora de las pruebas PISA, ha propuesto para sus distintos estados miembros un Marco Común de Competencia Digital. Muchos de estos enfoques proponen construir un modelo competencial que responda a las demandas de la economía y el mercado laboral (OCDE, 2016).

Entretanto, algunos países vienen generando sus propias definiciones, que enfatizan, dependiendo el caso, la dimensión tecnológica o productiva de los medios digitales o las capacidades informacionales. Por ejemplo, en el Perú el currículo nacional plantea desde el 2017 una competencia que aspira a que el estudiante aproveche "responsablemente las TIC para interactuar con la información, gestionar su comunicación y aprendizaje" (Ministerio de Educación del Perú [MINEDU], 2017) y en Chile el currículo para los estudiantes de séptimo básico a segundo medio (12 a 15 años) incluye como uno de sus objetivos generales que los estudiantes puedan "acceder a información y comunicarse usando las tecnologías de la información y la comunicación en forma reflexiva y eficaz" (Ministerio de Educación de Chile [Mineduc], 2015). Ante tanta variedad, conviene presentar la definición que consideramos más integradora y pertinente.

\subsection{Nuestra definición de competencia}

En el período 2005-2010, Joan Ferrés lideró un proyecto financiado en un primer momento por el Consejo del Audiovisual de Cataluña (CAC) y luego por el Ministerio de Educación de España, que tenía como objetivo evaluar la competencia mediática de la ciudadanía española. Además de situar una línea base acerca de la competencia mediática de los ciudadanos a nivel nacional, el estudio tuvo el mérito de definir dimensiones e indicadores para medir esta competencia, que se han convertido en un referente al momento de investigar la educación mediática en Iberoamérica (Ferrés 2006, 2007). 
Posteriormente, el 2012, Ferrés y Piscitelli propusieron una nueva versión de la competencia a partir de la existente. Esta propuesta fue consensuada con 50 expertos de Iberoamérica y se organizó en torno a seis dimensiones: lenguajes, tecnología, procesos de interacción, procesos de producción y difusión, ideología y valores y estética (Tabla 2). La competencia mediática propuesta por los autores fue definida como la "capacidad de interactuar de manera crítica con mensajes mediáticos producidos por los demás, y siendo capaz de producir y de diseminar mensajes propios" (pp. 77-78). Esta capacidad incluye dentro de sus indicadores el descubrir la manera en que las representaciones mediáticas estructuran nuestra percepción de la realidad y evaluar la fiabilidad de las fuentes de información.

Al mismo tiempo, la propuesta fue estructurada en torno a dos ámbitos de trabajo: el de la producción de mensajes propios y el de la interacción con mensajes ajenos. La idea de los autores fue desarrollar esta competencia en el contexto de una cultura participativa, que compagine el espíritu crítico y estético con la capacidad expresiva y el desarrollo de la autonomía personal con el compromiso social y cultural. Asimismo, motivar un diálogo más cercano entre las revoluciones tecnológica y neurobiológica, asumiendo los cambios producidos en la concepción de la mente humana, sobre todo en lo referente al peso de las emociones y del inconsciente sobre los procesos razonados y conscientes, que usualmente no son parte de las propuestas de competencia mediática.

Tabla 1. Resumen de la propuesta articulada de dimensiones de la competencia mediática de Ferrés y Piscitelli

\begin{tabular}{|c|c|}
\hline Dimensión & Indicadores (resumen) \\
\hline Lenguajes & $\begin{array}{l}\text { Conocimiento de los códigos, capacidad para utilizarlos y para analizar mensajes } \\
\text { escritos y audiovisuales desde la perspectiva del sentido y el significado, de las } \\
\text { estructuras narrativas y de las categorías y géneros. }\end{array}$ \\
\hline Uso de Tecnología & $\begin{array}{l}\text { Conocimiento y capacidad de utilización de las herramientas que hacen posible la } \\
\text { comunicación escrita y audiovisual para entender cómo se elaboran los mensajes. } \\
\text { Comprensión del papel que desempeñan las TIC en la sociedad. }\end{array}$ \\
\hline $\begin{array}{l}\text { Procesos de } \\
\text { interacción }\end{array}$ & $\begin{array}{l}\text { Capacidad de valorar, seleccionar, revisar y autoevaluar la propia dieta mediática. } \\
\text { Capacidad de valorar críticamente los elementos cognitivos, racionales, } \\
\text { emocionales y contextuales que intervienen en el intercambio de mensajes. }\end{array}$ \\
\hline $\begin{array}{l}\text { Procesos de } \\
\text { producción y difusión }\end{array}$ & $\begin{array}{l}\text { Conocimiento de las funciones y tareas de los agentes de producción, las fases de } \\
\text { los procesos de producción y difusión y los códigos de regulación. Capacidad para } \\
\text { elaborar, seleccionar, compartir y diseminar mensajes mediáticos. }\end{array}$ \\
\hline Ideología & $\begin{array}{l}\text { Capacidad de lectura comprensiva y crítica, de análisis crítico y actitud de } \\
\text { selección de los mensajes mediáticos, en cuanto representaciones de la realidad. }\end{array}$ \\
\hline Estética & $\begin{array}{l}\text { Capacidad de analizar y de valorar los mensajes audiovisuales desde el punto de } \\
\text { vista de la innovación formal y temática y la educación del sentido estético. }\end{array}$ \\
\hline
\end{tabular}

Fuente: Mateus y Suárez-Guerrero (2017) basado en Ferrés y Piscitelli (2012) 


\section{Análisis de tres experiencias: España, Chile y Perú}

\subsection{Metodología}

A partir de los parámetros indicados y la definición anterior se realizaron en los ultimos años diversas experiencias de aplicación de instrumentos de evaluación de competencia mediática en algunos países de Iberoamérica. A continuación, analizaremos, a partir de la revisión de fuentes secundarias, tres experiencias de mediciones recientes llevadas a cabo en España, Chile y Perú, con el objetivo de identificar los problemas metodológicos detectados y proponer en el último apartado algunos retos para superarlos.

\subsection{España}

Entre 2009 y 2010 se aplicó un cuestionario de la competencia mediática basado en el constructo presentado en el punto anterior a 6,626 personas de 17 comunidades autónomas de España como parte de un proyecto I+D que incluyó a universidades e institutos de todas las comunidades. Se dividió en dos partes. En la primera se realizaron preguntas genéricas para identificar las características referentes al nivel de estudio, sexo y edad del entrevistado, mientras que en la segunda se evaluaron las dimensiones de la competencia mediática a través de 28 preguntas. El cuestionario fue administrado en papel, con un monitor, y las salas debían estar equipadas con un ordenador con CD o DVD que permitiera reproducir dos spots publicitarios. Luego del cuestionario se aplicaron grupos de discusión y entrevistas grupales.

El diseño del cuestionario partió de la premisa de que las competencias en educación mediática no se evalúan porque no se enseñan, pero que "probablemente no se enseñan porque, al no ser evaluadas, no hay conciencia de las carencias que existen en este ámbito" (Ferrés et al., 2011, p. 11). Se presentó, por lo anterior, como un instrumento pionero.

Entre sus resultados, el estudio mostró que en la mayoría de las seis dimensiones de la competencia mediática los españoles no obtuvieron los niveles mínimos aprobatorios. Las peores puntuaciones estuvieron en las dimensiones estética y de lenguajes, mientras que los mejores fueron en el uso de tecnología, en especial entre los más jóvenes.

Además de los resultados, los autores del proyecto expusieron las dificultades para diseñar un instrumento que evaluara las dimensiones y los indicadores, la inclusión/exclusión de preguntas, la codificación y la fiabilidad de los resultados por cada dimensión. La falta de referentes respecto a la medición de la competencia mediática produjo un alto número de opiniones y disensos respecto a los criterios y herramientas de evaluación de las dimensiones. Especial dificultad tuvo la elaboración de ejercicios visuales, dado que las pruebas piloto que se realizaron mostraron que este tipo de preguntas debían ser revisadas, lo que implicó una gran cantidad de tiempo hasta encontrar los interrogantes definitivos.

El cuestionario tuvo algunos problemas particulares que se evidenciaron en su validación. Entre ellos, algunas preguntas con resultados extremos: los rendimientos bajos se justificaron porque la ciudadanía no había tenido formación en este ámbito, en especial en lo referido a las emociones; mientras que los rendimientos altos se explicaron por la intencionalidad de incluir preguntas con baja dificultad para mostrar que un porcentaje significativo de las personas tenías carencias básicas, lo que ayudaría a justificar la necesidad de educación mediática en el país.

Las pruebas piloto permitieron determinar que se debían eliminar las preguntas abiertas. No obstante, se mantuvieron dos referidas a la valoración estética de un spot, debido a la dificultad de medir esta dimensión. También se incluyeron preguntas que no puntuaban, con el fin de darle una mayor confianza a la persona que responde y lograr que venciera las reticencias ante un tema en el que no ha sido formada. 
En cuanto a la validación y la fiabilidad, el instrumento es adecuado para evaluar la competencia mediática en general, no para cada una de las dimensiones en particular. Además, hay dimensiones que tienen un mayor peso, como la de la Ideología y los Valores, en contraposición a otras, como la de producción y programación o la dimensión estética, que solo cuenta con dos preguntas, como ya dijimos.

A pesar de estas dificultades, la medición de la competencia adquiere validez científica debido a la representatividad de la muestra y a que los datos fueron complementados con una segunda parte cualitativa, en la que se realizaron entrevistas y grupos de discusión en algunas comunidades españolas. El estudio aportó una línea base acerca de la competencia mediática a nivel nacional y se constituyó en un referente para evaluar la competencia mediática en Iberoamérica.

\subsection{Chile}

En el caso de Chile, en 2016 se adaptó un cuestionario de educación mediática utilizado en el proyecto La Competencia en Comunicación Audiovisual en un Entorno Digital. Diagnóstico de Necesidades en Tres Ámbitos Sociales (2011-2014) en el ámbito de la enseñanza obligatoria, específicamente el que iba dirigido a los profesores.

En España el cuestionario tuvo cuatro versiones: infantil, primaria, secundaria y docentes. Respecto de este último, el instrumento contaba con 43 preguntas, de las que cinco eran de identificación personal y sobre el establecimiento escolar y 38 referidas a las dimensiones de la competencia mediática propuestas por Ferrés en 2006. Entre estas últimas se incluían cuestiones sobre el uso didáctico de los medios y las tecnologías (Pérez-Rodríguez, DelgadoPonce y García-Ruiz, 2015).

El instrumento incluía 13 preguntas en escala de Likert en las que el docente señalaba el grado de acuerdo que tiene sobre su competencia adquirida, y 15 preguntas en las que se preguntaba sobre conocimientos generales en torno a los medios de comunicación en escala dicotómica, como vemos en la Tabla 2. El resto de las preguntas incluía ítems de opción múltiple.

Los resultados de fiabilidad del cuestionario fueron mostrados de forma separada, debido a que incluía respuestas de autopercepción mediante escala Likert y dicotómicas. Los autores reportaron que la fiabilidad de los ítems dicotómicos fue moderada, mientras que la obtenida para los ítems de autopercepción resultó mayor o alta (González, Gozalvez y Ramírez, 2014, p. 128).

Tabla 2. Respuestas del Cuestionario Educación Mediática para Profesores

\begin{tabular}{|c|c|c|}
\hline Pregunta & Opciones de Respuesta & Categoría respuesta \\
\hline $\begin{array}{l}\text { En los mensajes de los medios (incluido Internet), } \\
\text { distingo los diferentes códigos (verbal, icónico, } \\
\text { musical...) y los registros (formal, informal, culto, } \\
\text { coloquial...) utilizados por el emisor. }\end{array}$ & $\begin{array}{ll}\text { 1. } & \text { No } \\
\text { 2. } & \text { Un poco } \\
\text { 3. } & \text { Bastante } \\
\text { 4. } & \text { Mucho }\end{array}$ & Autovaloración \\
\hline $\begin{array}{l}\text { Google, Safari, Bing o Yahoo!... ¿Son buscadores de } \\
\text { Internet exentos de publicidad comercial? }\end{array}$ & $\begin{array}{ll}\text { 1. } & \text { Sí } \\
\text { 2. } & \text { No } \\
\text { 3. } & \text { No lo sé }\end{array}$ & Conocimiento \\
\hline
\end{tabular}

Fuente: Universidad de Huelva, 2010. 
El cuestionario para la evaluación de docentes fue adaptado para ser aplicado a estudiantes de los últimos años de la carrera de educación parvularia, es decir, a futuras docentes del nivel infantil. La adaptación partió de las dimensiones de la competencia mediática propuesta por Ferrés (2007) y revisadas por Ferrés y Piscitelli (2012). Esta revisión incorporó las dimensiones de los procesos de interacción y de la producción y la difusión, en reemplazo de las dimensiones de la recepción e interacción y de la producción y programación.

El cuestionario se aplicó a una muestra de 100 estudiantes de ocho universidades. Posteriormente se realizaron cuatro grupos de discusión (dos en regiones y dos en la capital). El cuestionario está conformado por 42 preguntas, cuatro correspondientes a la autopercepción de la formación en educación mediática, una sobre datos sociodemográficos (edad, universidad, sexo, ciudad), tres respecto a los recursos tecnológicos con que se cuenta en la universidad; cuatro referidas a su opinión en torno a la didáctica de la educación mediática en la universidad y en jardines infantiles, y 29 para evaluar la competencia mediática.

La actualización de las dimensiones fue una primera dificultad del instrumento utilizado en Chile, puesto que en el cuestionario original se utilizaron las primeras dimensiones de la competencia mediática (2006, 2007). Un ejemplo de esta adaptación lo vemos en la Tabla 3.

Tabla 3. Adaptación a las Dimensiones de la Competencia Mediática actualizadas

\begin{tabular}{|l|l|l|l|}
\hline & \multicolumn{1}{|c|}{ Pregunta } & Dimensión & \multicolumn{1}{|c|}{ Indicador } \\
\hline $\begin{array}{l}\text { Cuestionario } \\
\text { Original }\end{array}$ & $\begin{array}{l}\text { Me relaciono a través de } \\
\text { los medios con colegas y } \\
\text { profesionales del ámbito } \\
\text { educativo }\end{array}$ & $\begin{array}{l}\text { Recepción e } \\
\text { interacción }\end{array}$ & $\begin{array}{l}\text { Saber si tienen la capacidad de } \\
\text { interaccionar con personas diversas en } \\
\text { entornos cada vez más plurales y } \\
\text { multiculturales. }\end{array}$ \\
\hline $\begin{array}{l}\text { Cuestionario } \\
\text { Chile }\end{array}$ & $\begin{array}{l}\text { ¿Me relaciono a través de } \\
\text { los medios de } \\
\text { comunicación con otras } \\
\text { (os) educadoras (es) en } \\
\text { formación y } \\
\text { educadoras(es) en } \\
\text { ejercicio? }\end{array}$ & $\begin{array}{l}\text { Procesos de } \\
\text { Interacción }\end{array}$ & $\begin{array}{l}\text { Capacidad de llevar a cabo un trabajo } \\
\text { colaborativo mediante la conectividad } \\
\text { y la creación de plataformas que } \\
\text { facilitan las redes sociales. }\end{array}$ \\
\hline
\end{tabular}

Fuente: Elaboración propia

Una segunda complicación fue adaptar el lenguaje al contexto chileno y revisar la pertinencia de las preguntas referidas a la tecnología. Lo segundo implica un desafío respecto a preguntas muy específicas que pierden validez con el tiempo debido al desarrollo tecnológico. Una tercera dificultad fue referida al análisis. Surgieron los siguientes interrogantes: ¿Cada pregunta debe medir solo un indicador de una dimensión o se puede medir más de uno? Si es lo primero, ¿es posible aislar cada aspecto de una dimensión (indicador)? Si es lo segundo, ¿sigue siendo válido un cuestionario en el que las preguntas miden más de una dimensión? Si la respuesta es afirmativa, ¿se pueden seguir comparando las dimensiones? Para complicar más lo anterior, encontramos preguntas que se ajustaban bastante a dos indicadores (Tabla 4).

Finalmente, se optó porque cada pregunta midiera de manera aislada un indicador de una dimensión de la competencia mediática. Esta alternativa se tomó para facilitar el análisis, porque, como hemos señalado, las dimensiones del cuestionario original no eran las mismas que en su versión adaptada, y debido a que las respuestas presentaban una dificultad de comparación por tener opciones de autovaloración en escala de Likert y dicotómicas. 
Tabla 4. Preguntas que se ajustan a más de un indicador

\begin{tabular}{|c|c|c|}
\hline Pregunta & Dimensión & Indicador \\
\hline $\begin{array}{l}\text { He usado en alguna ocasión los medios y } \\
\text { tecnologías comunicativas para... (puede } \\
\text { marcar más de una casilla) } \\
\checkmark \quad \text { Comprobar la corrección de mis } \\
\text { datos en las listas electorales } \\
\checkmark \quad \text { Votar electrónicamente en alguna } \\
\text { encuesta } \\
\checkmark \quad \text { Unirme a algún partido político o a } \\
\text { alguna campaña política } \\
\checkmark \quad \text { Transmitir o cursar una queja ante } \\
\text { el ayuntamiento o autoridad pública }\end{array}$ & $\begin{array}{l}\text { Procesos de } \\
\text { Interacción } \\
\text { expresión }\end{array}$ & $\begin{array}{l}\text { Actitud activa en la interacción } \\
\text { con las pantallas, entendidas } \\
\text { como oportunidad para construir } \\
\text { una ciudadanía más plena, un } \\
\text { desarrollo integral, para } \\
\text { transformarse y para transformar } \\
\text { el entorno. }\end{array}$ \\
\hline $\begin{array}{l}\checkmark \quad \text { Participar en alguna acción de } \\
\text { protesta o manifestación colectiva de } \\
\text { interés público. } \\
\checkmark \quad \text { Participar en alguna asociación } \\
\text { solidaria u ONG } \\
\checkmark \quad \text { Boicotear algún producto o servicio } \\
\checkmark \quad \text { Dar mi opinión en alguna } \\
\text { plataforma con fines sociales o políticos }\end{array}$ & $\begin{array}{c}\text { Ideología y Valores } \\
\text { Análisis. }\end{array}$ & $\begin{array}{l}\text { Saber la capacidad de aprovechar } \\
\text { las nuevas herramientas } \\
\text { comunicativas para transmitir } \\
\text { valores y contribuir a la mejora } \\
\text { del entorno, desde una actitud de } \\
\text { compromiso social y cultural, } \\
\text { conocernos mejor y para abrirnos } \\
\text { a otras experiencias. }\end{array}$ \\
\hline
\end{tabular}

Fuente: Elaboración propia

A partir de esta opción se pudo dicotomizar las preguntas y comparar los resultados entre las dimensiones. Es decir, se transformaron todas las respuestas a dos valores, negativo y positivo. Las dimensiones que tuvieron una mayor correlación fueron las de uso de tecnología con procesos de interacción, y las la ideología y valores con las de procesos de interacción y con estética.

Identificamos como una de las dificultades que, más que medir la competencia mediática, se mide la autopercepción de la competencia. Esto ocurre en al menos 13 preguntas, que tienen como respuesta una escala Likert con la autovaloración ante una situación.

\subsection{Perú}

Para el caso peruano, el 2017 se adaptó, al igual que en el caso chileno, el constructo propuesto por Ferrés y Piscitelli (2012) a un cuestionario de autopercepción que fue aplicado a docentes en formación. El interés de este trabajo radicó en conocer hasta qué punto los estudiantes de educación de distintas instituciones se sentían competentes mediáticamente, pues no existe un diagnóstico similar en el país.

La evaluación de capacidades a partir de la autopercepción del individuo se fundamenta en la teoría cognitiva social, desarrollada, entre otros autores, por Bandura (1977), y sostiene que las personas somos seres proactivos $\mathrm{y}$, como tales, desarrollamos la capacidad de regularnos, reflexionar y organizarnos a nosotros mismos. Así, nuestro sistema interno influye directamente en el logro de las metas que nos trazamos: si no nos creemos capaces de cumplir cierta tarea, ni siquiera la intentaremos; por el contrario, tampoco la sola creencia nos asegura el cumplimiento, pues se requieren determinadas aptitudes, por lo que la misma teoría plantea limitaciones. No obstante, en el campo docente se ha hallado que, además de conocimientos y 
habilidades, los docentes deben creerse capaces de lograr buenos resultados, por lo que el concepto de autoeficacia docente adquiere un valor especial en la investigación educativa (Mahler, Großschedl y Harms 2017).

Como procedimiento para conocer el grado de competencia mediática autopercibida en los estudiantes de educación, convertimos los indicadores del constructo teórico de seis dimensiones a treinta enunciados adaptados a situaciones docentes --que fueron validados con un pretest y juicio de expertos-- (ver Tabla 4). Cada afirmación fue valorada por los participantes utilizando una escala de Likert de cinco puntos en relación con el grado de acuerdo o desacuerdo.

Tabla 5. Ejemplos de adaptación de enunciados del cuestionario aplicado a estudiantes de educación en el Perú

\begin{tabular}{|c|c|c|}
\hline Dimensión & Indicadores del constructo & Ítems del cuestionario \\
\hline Lenguajes & $\begin{array}{l}\text { Capacidad de interpretar y de valorar los } \\
\text { diversos códigos de representación y la } \\
\text { función que cumplen en un mensaje. }\end{array}$ & $\begin{array}{l}\text { Soy capaz de utilizar materiales didácticos } \\
\text { procedentes de los medios explotando } \\
\text { sus propios códigos }\end{array}$ \\
\hline Tecnologías & $\begin{array}{l}\text { Comprensión del papel que desempeñan } \\
\text { en la sociedad las tecnologías de la } \\
\text { información y de la comunicación y de sus } \\
\text { posibles efectos. }\end{array}$ & $\begin{array}{l}\text { Soy capaz de reconocer riesgos } \\
\text { tecnológicos latentes del entorno digital } \\
\text { (correos fraudulentos, software } \\
\text { malicioso, etc.) }\end{array}$ \\
\hline Interacción & $\begin{array}{l}\text { Conocimiento de las posibilidades legales } \\
\text { de reclamación ante el incumplimiento de } \\
\text { las normas vigentes en materia } \\
\text { audiovisual, y actitud responsable ante } \\
\text { estas situaciones. }\end{array}$ & $\begin{array}{l}\text { Soy capaz de presentar una queja formal } \\
\text { por algún contenido mediático que } \\
\text { considere ofensivo ante las instancias } \\
\text { correspondientes }\end{array}$ \\
\hline Estética & $\begin{array}{l}\text { Sensibilidad para reconocer una } \\
\text { producción mediática que no se adecue a } \\
\text { unas exigencias mínimas de calidad } \\
\text { estética. }\end{array}$ & $\begin{array}{l}\text { Soy capaz de reconocer una producción } \\
\text { audiovisual formalmente deficiente (por } \\
\text { la calidad de imagen, sonido o montaje) }\end{array}$ \\
\hline $\begin{array}{l}\text { Ideología y } \\
\text { valores }\end{array}$ & $\begin{array}{l}\text { Capacidad de evaluar la fiabilidad de las } \\
\text { fuentes de información, extrayendo } \\
\text { conclusiones críticas tanto de lo que se } \\
\text { dice como de lo que se omite. }\end{array}$ & $\begin{array}{l}\text { Soy capaz de contrastar la fiabilidad de la } \\
\text { información que obtengo en medios } \\
\text { digitales. }\end{array}$ \\
\hline $\begin{array}{l}\text { Producción y } \\
\text { difusión }\end{array}$ & $\begin{array}{l}\text { Conocimiento de los factores que } \\
\text { convierten las producciones corporativas } \\
\text { en mensajes sometidos a los } \\
\text { condicionamientos socioeconómicos de } \\
\text { toda industria. }\end{array}$ & $\begin{array}{l}\text { Soy capaz de advertir conflictos que se } \\
\text { puedan crear entre los intereses de los } \\
\text { propietarios de un medio, los que lo } \\
\text { financian y la información que emite. }\end{array}$ \\
\hline
\end{tabular}

Fuente: Elaboración propia

El cuestionario se aplicó a una muestra de 501 estudiantes de educación de cuatro instituciones de Lima, y se complementaron los resultados cuantitativos con cuatro grupos de discusión (uno en cada institución participante) (Mateus, Hernández-Breña y Figueras-Maz, 2019). El cuestionario está conformado por 36 ítems, seis correspondientes a la información demográfica 
(edad, género, universidad, especialidad de la carrera, años de estudio y lugar de nacimiento) y el resto estuvieron organizados con cinco enunciados para cada una de las seis dimensiones de la competencia, que debían valorar en una escala de Likert de cinco grados ( 1 = "totalmente en desacuerdo" y 5 = "totalmente de acuerdo").

La validación estadística del instrumento fue muy positiva (alfa de Cronbach $=0.943$ ), y quedó muy claro el alto grado de correlación entre todas las variables $\left({ }^{*} \mathrm{p}<0.01\right.$ en todas las dimensiones). Los resultados, asimismo, mostraron una confianza alta de los futuros docentes hacia sus propias capacidades mediáticas (media $=3,57, \mathrm{SD}=0,60$ ), aunque se registraron ligeras diferencias en los rendimientos cuando comparamos las dimensiones. La dimensión mejor valorada es la de procesos de interacción (que incluye capacidades como búsqueda de información y elaboración de contenidos digitales) y la peor fue la de producción (con preguntas sobre aspectos técnicos como los roles en la realización de una película o la diferencia entre un medios públicos y privados).

En cuanto a las dificultades detectadas, destacamos la del desconocimiento genérico de la educación mediática como concepto. En una evaluación cualitativa complementaria, muchos participantes tenían serios problemas en definir conceptos básicos como TIC. Esto supuso, como conclusión, que en buena medida el cuestionario cumpliera un fin formativo, pues les permitió a los participantes hacerse una idea de lo que era la competencia mediática y su complejidad. No obstante, para no haber recibido formación explícita, el rendimiento reportado fue curiosamente alto, lo que nos devuelve a la discusión de los límites de la autopercepción.

La adaptación de los indicadores propuestos por Ferrés y Piscitelli a capacidades concretas que los docentes peruanos pudieran identificar supuso, también, un reto cultural: muchos de los indicadores apelan a situaciones que son diferentes en cada país (por ejemplo, las leyes o las instituciones a cargo de velar por la calidad de los medios), además de que las referencias tecnológicas deben ser constantemente actualizadas. Asimismo, una dificultad de análisis fue causada por el alto nivel de correlación reportada entre todas las variables, lo que nos llevó a preguntarnos si cada dimensión tiene autonomía (para comparar, por ejemplo, en qué dimensión rinden más o menos) o si la competencia debe ser evaluada siempre de manera integrada (dado que todos los ítems se relacionan con más de una dimensión).

\subsection{A modo de balance}

En el recuento anterior hemos identificado algunos entrampamientos metodológicos que vale la pena resumir. Por un lado, la calidad autoperceptiva de los instrumentos, si bien aporta al diagnóstico, no debe ser confundida como una medida fiable de la capacidad real de los participantes para interactuar críticamente con los medios. Los resultados en ocasiones extremos reportados en el caso español dan cuenta de esto. Por el otro, la dificultad para medir las dimensiones de la competencia mediática de forma aislada, dado que las fronteras entre las capacidades son muy finas, genera una alta correlación entre todas. Esto nos invita a cuestionar la posibilidad de medir las dimensiones de manera interdependiente.

\section{Discusión y retos a futuro}

El problema para medir la competencia mediática, como hemos señalado, tiene tres niveles altamente correlacionados: el político, el pedagógico y el metodológico, que configuran, en la actualidad, un círculo vicioso. En cuanto al problema político, la competencia mediática tiene el reto de hacerse un espacio en las agendas educativas que trascienda la noción de competencias digitales como un repertorio de habilidades operativas vinculadas a tecnologías 
que sirven para mejorar la fuerza laboral, y se reconozca como un derecho para el ejercicio de la ciudadanía.

En el terreno pedagógico, al no existir una definición unívoca de la competencia mediática a nivel internacional, su presencia no está formalizada en todos los currículos nacionales, a diferencia de otras áreas de conocimiento más tradicionales, como la matemática, donde se conoce perfectamente cuáles son los contenidos y progresiones del aprendizaje (es universal que la suma es previa a la resta y la aritmética previa al álgebra, por ejemplo). En consecuencia, no es posible comparar la evaluación de la competencia en el ámbito internacional y resulta obvia la existencia de múltiples instrumentos creados para este fin, sin que ofrezcan siempre una definición precisa o unánime. Para el desarrollo de una política educativa que atienda las competencias mediáticas (que incluyen, pero no se agotan, en las llamadas competencias digitales e informacionales) es preciso desarrollar modelos competenciales e instrumentos que partan de dimensiones claramente establecidas, pues no podremos mejorar la competencia de los ciudadanos si no podemos diagnosticarla.

A nivel metodológico, a pesar de los avances que se han realizado en los últimos años, la mayoría de los instrumentos que se utilizan para medir las competencias de la población utilizan preguntas de autopercepción. Este tipo de preguntas permite acercarnos a conocer lo que las personas creen saber, pensar o sentir, y tiene una importancia teórica antes referida, pero no nos asegura conocer lo que realmente saben, piensan y sienten. Si bien existe teoría suficiente que da cuenta de la importancia de este tipo de cuestionarios, creemos que no son suficientes para evaluar las capacidades en el plano concreto de la interacción con los medios, que es lo que buscamos en nuestra propuesta.

Una revisión de 88 estudios que evaluaban la competencia mediática en distintos países concluyó que al menos una cuarta parte de ellos exhiben problemas de validez de contenido (Potter y Thai, 2016). En este sentido, es vital proporcionar una conceptualización clara de la competencia y de las dimensiones que la componen. Por ejemplo, definir la competencia mediática como habilidades de producción y conocimientos en torno a los medios supone que los indicadores de ambas capacidades estén concretamente establecidos y sean presentados de manera equilibrada.

\subsection{Retos y alternativas posibles}

El reto identificado es la construcción de un cuestionario que logre atender algunos de los entrampamientos señalados en las experiencias previas de aplicación. A partir de nuestra experiencia en el campo, estructuramos una propuesta en torno a dos ideas: que sea integradora en cuanto a los contenidos e innovadora en cuanto a la metodología.

Hemos dado cuenta de un predominio de la evaluación de contenidos que se reducen a la dimensión del uso de la tecnología, ante lo cual pensamos que es fundamental ampliar los contenidos a seis dimensiones que den cuenta de lo mediático como un fenómeno multidimensional e integral, donde se incluye lo informacional, lo digital y también el entretenimiento (Ferrés y Piscitelli, 2012).

La integración de múltiples contenidos también se refiere a incorporar las innovaciones procedentes de la aparición de nuevas tecnologías y de nuevas prácticas comunicativas, así como también de los conocimientos que hoy tenemos a partir de los descubrimientos de la neurociencia en torno a la mente que interacciona con las tecnologías de la comunicación. Al respecto, buscaremos una evaluación que atienda a la idea de prosumidor o de emirec, es decir, de un consumidor o receptor de medios que al mismo tiempo se expresa con ellos, sacando 
partido de las posibilidades que ofrecen las tecnologías para que el ciudadano pueda producir mensajes en la misma medida que consumirlos.

La innovación metodológica tiene como principio superar los planteamientos autoperceptivos. Como vimos, gran parte de las propuestas de evaluación de competencia digital, informacional y mediática que se encuentran se basan en parámetros meramente declarativos. Las preguntas deben proponer un reto, desafiando a la persona tanto racional como emocionalmente. Le debe permitir mostrar que sabe resolver un problema antes que demostrar la memorización de contenidos. En otros casos debería buscarse un grado de reflexión que permita demostrar su competencia más allá de un caso concreto.

Las preguntas desafiantes y reflexivas se lograrán a través de una herramienta interactiva que dé cuenta de los últimos avances en el ámbito tecnológico, puesto que para evaluar las habilidades ante los medios se deben, precisamente, evaluar las posibilidades de los mismos.

Con todo ello, creemos que se conseguiría un salto cualitativo en la evaluación de las competencias digital, informacional y mediática, lo cual garantizaría mayores dosis de objetividad. Los resultados de un instrumento como el referido deberían permitir la elaboración de estudios diagnóstico a gran escala, así como análisis comparativos que den luces a los decisores de políticas y proyectos educativos sobre la conveniencia de trabajar la educación mediática y su correspondiente competencia, como un conjunto de capacidades fundamentales para los ciudadanos de este siglo. Resultados que servirían como base y punto de comparación para, en un segundo momento, en una fase cualitativa, profundizar en las características y en la pertinencia local de la competencia mediática.

\section{Bibliografía}

Buitrago, A. (2016). Los profesionales de la comunicación ante la competencia mediática. Segovia: Universidad de Valladolid. Tesis para optar al grado de doctor por la Universidad de Valladolid.

Comisión Europea (2018). A multi-dimensional approach to disinformation. Report of the independent High level Group on fake news and online disinformation. Recuperado de: http://ec.europa.eu/newsroom/dae/document.cfm?doc_id=50271

De Ketele, J. (2008). Enfoque socio-histórico de las competencias en la enseñanza. Profesorado. Revista de Currículum y Formación de Profesorado, 12 (3), 1-12. Recuperado de: http://www.redalyc.org/pdf/567/56712875002.pdf.

Ferrés, J. (2006). La competència en comunicació audiovisual: proposta articulada de dimensions i indicadors. Quaderns del CAC, 25. 9-17.

Ferrés, J. (2007). La competencia en comunicación audiovisual: dimensiones e indicadores. Comunicar, 29. 100-107. https://doi.org/10.3916/C29-2007-14

Ferrés, J; García Matilla, A.; Aguaded, I.; Fernández Cavia, J.; Figueras, M. y Blanes, M. (2011). Competencia Mediática. Investigación sobre el grado de competencia de la ciudadanía en España. Madrid: Ministerio de Educación.

Ferrés, J. y Piscitelli, A. (2012). La competencia mediática: propuesta articulada de dimensiones e indicadores. Comunicar, 38, 75-82. https://doi.org/10.3916/C38-2012-02-08 
González Fernández, N., Gozálvez Pérez, V. y Ramírez García, A. (2015). La competencia mediática en el profesorado no universitario. Diagnóstico y propuestas formativas. Revista de Educación 367. 117-146 https://doi.org/10.4438/1988-592X-RE-2015-367-285

Guzmán Marín, F (2017). Problemática general de la educación por competencias. Revista Iberoamericana de Educación, 74(1), 107-120.

Hjarvard, S. (2016). Mediatización: La lógica mediática de las dinámicas cambiantes de la interacción social. La Trama de la Comunicación, 20 (1), pp. 235-252. Recuperado de: http://www.scielo.org.ar/pdf/trama/v20n1/v20n1a13.pdf

Mahler, D., Großschedl, J. y Harms, U. (2017). Opportunities to Learn for Teachers' SelfEfficacy and Enthusiasm. Education Research International, 2017, 1-17. https://doi. org/10.1155/2017/4698371

Mateus, J. C. y Suárez-Guerrero, C. (2017). La competencia TIC en el nuevo currículo peruano desde la perspectiva de la educación mediática. EDMETIC, 6(2), 129-147. https://doi. org/10.21071/edmetic.v6i2.6908

Mateus, J.C.; Hernández-Breña, W. y Figueras-Maz, M. (2019) Validation of a self-perceived media competence instrument for pre-service teachers. Culture and Education, 31(1). 1-29. https://doi.org/10.1080/11356405.2019.1597440

Ministerio de Educación, Cultura y Deporte de España (2018). Ley Orgánica para la Mejora de la Calidad Educativa. Competencia Digital. Recuperado de: http://www.mecd.gob.es/educacion/mc/lomce/el-curriculo/curriculo-primaria-esobachillerato/competencias-clave/digital.html

Ministerio de Educación del Perú (2017). Currículo Nacional de la Educación Básica. Recuperado de: http://www.minedu.gob.pe/curriculo/

Ministerio de Educación de Chile (2015). Bases Curriculares $7^{\circ}$ Básico a $2^{\circ}$ medio. Recuperado de: http://www.curriculumenlineamineduc.cl/605/articles-37136_bases.pdf

The Guardian (6 de mayo de 2014). OECD and Pisa tests are damaging education worldwide academics. Recuperado de https://www.theguardian.com/education/2014/may/06/oecd-pisatests-damaging-education-academics

Pérez-Rodríguez, M. A., Delgado-Ponce, Á., y García-Ruiz, R. (2015). Niños y jóvenes ante las redes y pantallas. Barcelona: Gedisa.

Potter, W. J. y Thai, C. (2016). Conceptual Challenges in Designing Measures for Media Literacy Studies. International Journal of Media and Information Literacy, (1-1), 27-42.

Scolari, C.A. (2018). Alfabetismo transmedia en la nueva ecología de los medios: libro blanco. Barcelona: Universitat Pompeu Fabra. Departament de Comunicació. Recuperado de: https://repositori.upf.edu/handle/10230/33910

Universidad de Huelva. (2010). Cuestionario sobre la competencia mediática. [Formulario en línea]. Recuperado de: http://www.uhu.es/competenciamediatica/ar/profesorado/ 